\title{
A New Wavelet Method for Solving a Class of Nonlinear Volterra-Fredholm Integral Equations
}

\author{
Xiaomin Wang \\ School of Engineering, Huazhong Agricultural University, Wuhan, Hubei 430070, China \\ Correspondence should be addressed to Xiaomin Wang; wangxm2012@mail.hzau.edu.cn
}

Received 1 March 2014; Accepted 4 August 2014; Published 28 August 2014

Academic Editor: Changbum Chun

Copyright (C) 2014 Xiaomin Wang. This is an open access article distributed under the Creative Commons Attribution License, which permits unrestricted use, distribution, and reproduction in any medium, provided the original work is properly cited.

\begin{abstract}
A new approach, Coiflet-type wavelet Galerkin method, is proposed for numerically solving the Volterra-Fredholm integral equations. Based on the Coiflet-type wavelet approximation scheme, arbitrary nonlinear term of the unknown function in an equation can be explicitly expressed. By incorporating such a modified wavelet approximation scheme into the conventional Galerkin method, the nonsingular property of the connection coefficients significantly reduces the computational complexity and achieves high precision in a very simple way. Thus, one can obtain a stable, highly accurate, and efficient numerical method without calculating the connection coefficients in traditional Galerkin method for solving the nonlinear algebraic equations. At last, numerical simulations are performed to show the efficiency of the method proposed.
\end{abstract}

\section{Introduction}

Integral equations have been found to be widely used in science and engineering. In recent years, the research on numerical solutions to various Volterra and Fredholm integral equations has been studied in both theoretical and practical fields [1-3] and has aroused a lot of interest.

We consider the following Volterra-Fredholm integral equation:

$$
\begin{aligned}
y(x)= & f(x)+\lambda_{1} \int_{a}^{h(x)} K_{1}(x, t) G_{1}(y(t)) d t \\
& +\lambda_{2} \int_{a}^{b} K_{2}(x, t) G_{2}(y(t)) d t,
\end{aligned}
$$

where $f(x), h(x), K_{1}(x, t)$, and $K_{2}(x, t)$ are known functions, $a \leq x, t \leq b, a \leq h(x)<\infty ; a, b$ are known constants; $y(x)$ is the unknown function. $G_{1}(y(t))$ and $G_{2}(y(t))$ are composite functions or nonlinear functions. It can be easily seen that, when $h(x)$ is a first-order polynomial, (1) is a functional integral equation with proportional delay.

Many approximate numerical methods have been suggested for solving Volterra-Fredholm equations. For example, collocation method is used for solving Volterra integral equations [4] and Volterra-Fredholm-type integral equations [5], respectively. Yalcinba and Sezer have employed the Taylor collocation method to solve second-order linear differential equations [6], linear integral differential equations [7], and Volterra-Fredholm integral equations [8]. Similar approaches of Chebyshev wavelet collocation method [9] and Haar wavelet collocation method [10] were used to solve this kind of integral equations. The continuous wavelet Galerkin method [11] and the Coiflet-Galerkin method [12] were proposed for the second kind integral equations and linear Volterra integral equations, respectively. Ren et al. [13] applied the Taylor polynomial method for a class of second kind integral equations. The Lagrange interpolation method $[14,15]$ was developed, by which the system of the linear integral equations was transformed into matrix equations via Lagrange collocation points. The spectral method [16] was presented for the Volterra integral equations, Fredholm integral equations, and Volterra-Fredholm integral equations, respectively. Although there are some effective methods above, it is still difficult to obtain a high accuracy numerical method which is suitable for nonlinear Volterra-Fredholm integral equations due to the existence of strong nonlinearity in these equations.

As a newly developed powerful mathematical tool, which has been developed mostly over the last twenty years, the wavelet has become widely used in the development 
of numerical schemes for solving differential and integral equations [17-20], Laplace inversions [21], and active vibration control of piezoelectric smart structures [22, 23]. In [11], Liang et al. solved the second kind integral equations by applying Galerkin method with continuous orthogonal wavelets, and one can find that using the Daubechies wavelets to solve the integral equation has almost the same numerical results as those of noncontinuous multiwavelets [24]. However, as pointed out in [10], few papers reported application of wavelet to solve the nonlinear Volterra-Fredholm integral equations.

In this study, we are concerned with the application of the Coiflet-type wavelet Galerkin method to the numerical solution of nonlinear Volterra-Fredholm integral equations. By combining techniques of modified Coiflet-type wavelet expansion and a single-point sampling of the function, we obtained an approximation scheme for a function defined on a bounded interval, which allows each expansion coefficient of nonlinear term being explicitly expressed. When applying this wavelet approximation scheme to the conventional Galerkin method, the original integral equation was transformed into the solving of some simple algebraic equations. The nonsingular property of the connection coefficients allows us not to calculate them in these algebraic equations and eventually develop a stable and efficient numerical method with high accuracy for solving nonlinear integral equations.

\section{Preliminaries}

For a function $f(x) \in \mathbf{L}^{2}(\mathbf{R})$, we have [25]

$$
f(x)=\lim _{n \rightarrow \infty} \sum_{k \in Z} c_{n, k} \phi_{n, k}(x) \approx \sum_{k \in Z} c_{n, k} \phi_{n, k}(x),
$$

where the expansion coefficients are

$$
c_{n, k}=\int_{\mathbf{R}} f(x) \phi_{n, k}(x) d x,
$$

where $\phi_{n, k}(x)=2^{n / 2} \phi\left(2^{n} x-k\right)$. By the generalized Gaussian integral method of wavelet proposed by Zhou and Wang [20], we have a good approximation of $c_{n, k}$ as follows:

$$
c_{n, k} \approx 2^{-n / 2} f\left(\frac{M_{1}+k}{2^{n}}\right),
$$

in which $M_{1}=\int_{-\infty}^{+\infty} x \phi(x) d x$ is the first-order moment of scaling function, which can be obtained accurately by filter coefficients in the two-scale equations [25]. The approximation accuracy of (4) depends on the corresponding wavelet function vanishing moment $\gamma$ and the reconstruction level $n$; it has been demonstrated that [20]

$$
\left|c_{n, k}-2^{-n / 2} f\left(\frac{M_{1}+k}{2^{n}}\right)\right| \leq M_{2} 2^{(-9 / 2) n},
$$

in which $M_{2}=5 / 384 \sup \left|d^{4} f(x) / d x^{4}\right|\left[2^{-n / 2}(3 \gamma-1)^{5}\right.$ $\left.\max |\phi(x)|+2(3 \gamma-1)^{4}\right]$. It can be seen from (5) that the approximation error decays as the reconstruction level $n$ increases. Substituting (4) into (2), we have

$$
f(x) \approx \sum_{k \in Z} f\left(\frac{M_{1}+k}{2^{n}}\right) \phi\left(2^{n} x-k\right) .
$$

The single-point reconstruction formula of function $f(x)$ in (6) has the following characteristics [20].

(1) For the composite function of the function $f(x)$, $\Pi[f(x)]$, its reconstruction (or approximation) formula is

$$
\Pi[f(x)] \approx \sum_{k \in Z} \Pi\left[f\left(\frac{M_{1}+k}{2^{n}}\right)\right] \phi\left(2^{n} x-k\right) .
$$

In this way, the importance of (7) is, for an arbitrary function $f(x)$ over the function operator $\Pi$ (which may be nonlinear operator) transformation, the computing of its right-hand side is equivalent to a linear operator computing. That is, the coefficient of the scaling function $\Pi[f(x)]$ can be obtained by putting operator $\Pi$ on the role of the coefficient $f(\cdot)$.

(2) For the integral operator or the derivative operator $\mathbf{D}$ on the function $f(x)$, we have

$$
\mathbf{D}[f(x)] \approx \sum_{k \in Z} f\left(\frac{M_{1}+k}{2^{n}}\right) \mathbf{D}\left[\phi\left(2^{n} x-k\right)\right] .
$$

That is, we just need to put the role of operator $\mathbf{D}$ on $\phi_{n, k}(x)$ in (8).

As we know, orthogonal scaling functions originally form a function basis on the whole real line. When the approximating function is defined only on a finite interval, one needs to truncate the wavelet series, which may introduce the boundary effect significantly and lead to reduced accuracy of the corresponding numerical method. Traditionally, general treatment of boundary conditions is by using the zeroextension, symmetric or periodic extension, and so on. To some extent, these approaches can effectively inhibit the jitter of the border when it is a special form of approximation function, but not universal. Different from these expansions of function, in this paper, we consider a natural extension treatment on the function by using Taylor series expansion at each boundary [25]. The extension can be smooth enough, and the corresponding boundary conditions can be explicitly embedded in the resulting scaling function expansions.

We assume that the function $g(x) \in \mathbf{L}^{2}[0, b]$, using Taylor series expansion on the borders, yields

$$
g(x)= \begin{cases}\sum_{i=0}^{M} \frac{1}{i !} \frac{d^{i} g(0)}{d x^{i}} x^{i} & x \in(-\delta, 0), \\ g(x) & x \in[0, b], \\ \sum_{i=0}^{M} \frac{1}{i !} \frac{d^{i} g(b)}{d x^{i}}(x-b)^{i} & x \in(b, b+\delta),\end{cases}
$$

in which $\delta>0, d^{i} g(0) / d x^{i}=\sum_{k=0}^{\alpha} p_{0, i, k} g\left(k / 2^{n}\right)$, and $d^{i} g(b) /$ $d x^{i}=\sum_{k=0}^{\alpha} p_{b, i, k} g\left(b-\left(k / 2^{n}\right)\right),(i=0,1,2, \ldots) ; p_{0, i, k}$ and 
$p_{b, i, k}$ are the numerical difference coefficients. In the present study, we take $\alpha=3, M=3$; according to four-point-Malkoff numerical difference formulas $[17,18,25]$, we know

$$
\begin{aligned}
& \mathbf{A}_{0}=\left(\begin{array}{cccc}
1 & 0 & 0 & 0 \\
-\frac{11}{6} & 3 & -\frac{3}{2} & \frac{1}{3} \\
2 & -5 & 4 & -1 \\
-1 & 3 & -3 & 1
\end{array}\right), \\
& \mathbf{A}_{1}=\left(\begin{array}{cccc}
1 & 0 & 0 & 0 \\
\frac{11}{6} & -3 & \frac{3}{2} & -\frac{1}{3} \\
2 & -5 & 4 & -1 \\
1 & -3 & 3 & -1
\end{array}\right)
\end{aligned}
$$

where $\mathbf{A}_{0}=\left\{2^{-i n} p_{0, i, k}\right\}$ and $\mathbf{A}_{1}=\left\{2^{-i n} p_{b, i, k}\right\},(i, k=0,1,2,3)$. For the specific boundary conditions, once the boundary conditions are given, in (10), certain elements of the matrix values will be adjusted. For example, for boundary conditions $x=0, d^{i} g(0) / d x^{i}=0$, we just need to set $p_{0, i, k}=0(k=$ $0,1,2,3)$ and keep all other elements $p_{0, j, k}(j \neq i)$ unchanged.

Then, (9) can be expressed as

$$
g(x)= \begin{cases}\sum_{k=0}^{3} g\left(\frac{k}{2^{n}}\right) T_{0, k}(x), & x \in(-\infty, 0) \\ g(x), & x \in[0, b] \\ \sum_{k=0}^{3} g\left(b-\frac{k}{2^{n}}\right) T_{b, k}(x), & x \in(b, \infty),\end{cases}
$$

where $T_{0, k}(x)=\sum_{i=0}^{M}\left(p_{0, i, k} / i !\right) x^{i}$ and $T_{b, k}=\sum_{i=0}^{M}\left(p_{b, i, k} / i !\right)(x-$ b) $)^{i}$.

Using the Coiflet-type scaling function with compact support set $[0,17](\gamma=6)$, for the scaling function adopted in this paper, we have that $M_{1}=7$; thus approximation equation (6) can be rewritten as

$$
g(x) \approx \sum_{k=2-3 N+M_{1}}^{b 2^{n}+M_{1}-1} g\left(\frac{k}{2^{n}}\right) \phi\left(2^{n} x+M_{1}-k\right) .
$$

Inserting (11) into (12) yields

$$
g(x) \approx \sum_{k=0}^{b 2^{n}} g_{k} \Phi_{b, n, k}(x),
$$

where $g_{k}=g\left(k / 2^{n}\right)$ and

$$
\begin{gathered}
\Phi_{b, n, k}(x) \\
=\left\{\begin{array}{cc}
\phi\left(2^{n} x+M_{1}-k\right) \\
+\sum_{j=2-3 N+M_{1}}^{-1} T_{0, k}\left(\frac{j}{2^{n}}\right) \phi\left(2^{n} x+M_{1}-j\right), \\
\phi\left(2^{n} x+M_{1}-k\right), & 4 \leq k \leq b 2^{n}-4, \\
\phi\left(2^{n} x+M_{1}-k\right) & 0 \leq k, \\
& +\sum_{j=1+b 2^{n}}^{n} T_{b, b 2^{n}-k}\left(\frac{j}{2^{n}}\right) \phi\left(2^{n} x+M_{1}-j\right),
\end{array}\right. \\
\quad b 2^{n}-3 \leq k \leq b 2^{n} .
\end{gathered}
$$

Thus, when specific boundary conditions are given, the corresponding coefficients of extension can be determined in accordance with the above process, and the corresponding improved scaling function is given by (14). It can be found that (14) is very convenient for dealing with nonlinear differential equations.

\section{Application}

In the following, we consider the solution of VolterraFredholm integral equation as shown in (1) by using the Coiflet-type wavelet Galerkin method proposed. For simplicity and without loss of generality, we take $h(x)=x, a=0$, and $b=1$ for traditional Volterra-Fredholm equation.

Let us rewrite (1) in the form

$$
y(x)=f(x)+\lambda_{1} V(x)+\lambda_{2} F(x),
$$

where $V(x)=\int_{0}^{x} K_{1}(x, t) G_{1}(y(t)) d t$ and $F(x)=\int_{0}^{1} K_{2}(x, t)$ $G_{2}(y(t))$.

Applying (13), we have

$$
\begin{aligned}
& y(x) \approx \sum_{k=0}^{2^{n}} y_{k} \Phi_{n, k}(x), \\
& f(x) \approx \sum_{k=0}^{2^{n}} f_{k} \Phi_{n, k}(x),
\end{aligned}
$$

where $x_{k}=k / 2^{n}, y_{k}=y\left(x_{k}\right)$, and $f_{k}=f\left(x_{k}\right)$. Similar to (7) and (8), one can approximate the nonlinear terms conveniently as

$$
\begin{aligned}
V(x) & \approx \sum_{k=0}^{2^{n}}\left[\sum_{j=0}^{2^{n}} K_{1}\left(x_{k}, t_{j}\right) G_{1}\left(y\left(t_{j}\right)\right) \Phi_{n, j}^{\int}\left(x_{k}\right)\right] \Phi_{n, k}(x) \\
& =\sum_{k=0}^{2^{n}} V_{k} \Phi_{n, k}(x), \\
F(x) & \approx \sum_{k=0}^{2^{n}}\left[\sum_{j=0}^{2^{n}} K_{2}\left(x_{k}, t_{j}\right) G_{2}\left(y\left(t_{j}\right)\right) \Phi_{n, j}^{\int}(1)\right] \Phi_{n, k}(x) \\
& =\sum_{k=0}^{2^{n}} F_{k} \Phi_{n, k}(x)
\end{aligned}
$$

in which $t_{j}=j / 2^{n}$, like

$$
\underbrace{\int \cdots \int}_{\Phi_{n, j}{ }^{\int \cdots \int}}\left(x_{k}\right)=\int_{0}^{x_{k}} \int_{0}^{\xi_{m}} \cdots \int_{0}^{\xi_{2}} \Phi_{n, j}(x) d x d \xi_{2} \cdots d \xi_{m} ;
$$


it can be obtained by [25]. Substituting (16)-(18) into (15), we have

$$
\begin{aligned}
\sum_{k=0}^{2^{n}} y_{k} \Phi_{n, k}(x) \approx & \sum_{k=0}^{2^{n}} f_{k} \Phi_{n, k}(x)+\lambda_{1} \sum_{k=0}^{2^{n}} V_{k} \Phi_{n, k}(x) \\
& +\lambda_{2} \sum_{k=0}^{2^{n}} F_{k} \Phi_{n, k}(x) .
\end{aligned}
$$

Multiplying both sides of (20) by the weight function $\Phi_{n, \xi}(x), \xi=0,1,2, \ldots, 2^{n}$, respectively, and taking integration over the interval $[0,1]$ yield

$$
\sum_{k=0}^{2^{n}} y_{k} \Gamma_{k, \xi} \approx \sum_{k=0}^{2^{n}} f_{k} \Gamma_{k, \xi}+\lambda_{1} \sum_{k=0}^{2^{n}} V_{k} \Gamma_{k, \xi}+\lambda_{2} \sum_{k=0}^{2^{n}} F_{k} \Gamma_{k, \xi}
$$

Here the connection coefficient $\Gamma_{k, \xi}$ can be obtained [25], since the matrix $\left\{\Gamma_{k, \xi}\right\}_{\left(2^{n}+1\right) \times\left(2^{n}+1\right)}$ is nonsingular, so we can further have

$$
y_{k} \approx f_{k}+\lambda_{1} V_{k}+\lambda_{2} F_{k}
$$

that is,

$$
\begin{aligned}
y_{k} \approx & f_{k}+\lambda_{1} \sum_{j=0}^{2^{n}} K_{1}\left(x_{k}, t_{j}\right) G_{1}\left(y\left(t_{j}\right)\right) \Phi_{n, j}^{\int_{0}^{x_{k}}}(t) \\
& +\lambda_{2} \sum_{j=0}^{2^{n}} K_{2}\left(x_{k}, t_{j}\right) G_{2}\left(y\left(t_{j}\right)\right) \Phi_{n, j}^{\int_{0}^{1}}(t) .
\end{aligned}
$$

By solving algebraic equation (23), which has $\left(2^{n}+1\right)$ equations, we can obtain the values of the unknown function $y_{k}, k=0,1,2, \ldots, 2^{n}$. Then, substituting them into (16), we can obtain the solution of (15).

\section{Numerical Experiments}

In this section we will give a series of numerical experimentsto illustrate the efficiency of the approaches in this paper.

Example 1. We consider the following integral equation given in [15]:

$$
y(x)=f(x)+\int_{0}^{x} K_{1}(x, t) y(t) d t-\int_{0}^{1} K_{2}(x, t) y(t) d t,
$$

where $K_{1}(x, t)=e^{x+t}, K_{2}(x, t)=e^{x+t}$, and $f(x)=e^{-x}-$ $e^{x}(x-1) \cdot y(x)=e^{-x}$ is the exact solution of this equation. It can be seen that the approximation solution obtained by the proposed method has a good agreement with the exact solution in Figure 1. Table 1 shows the absolute errors of this method under different resolution level $n$; the present absolute errors with 8 grid points are much smaller than those given by the Lagrange collocation method with 9 grid points [15]. And one can see that the absolute errors decrease quickly while the resolution level $n$ increases.

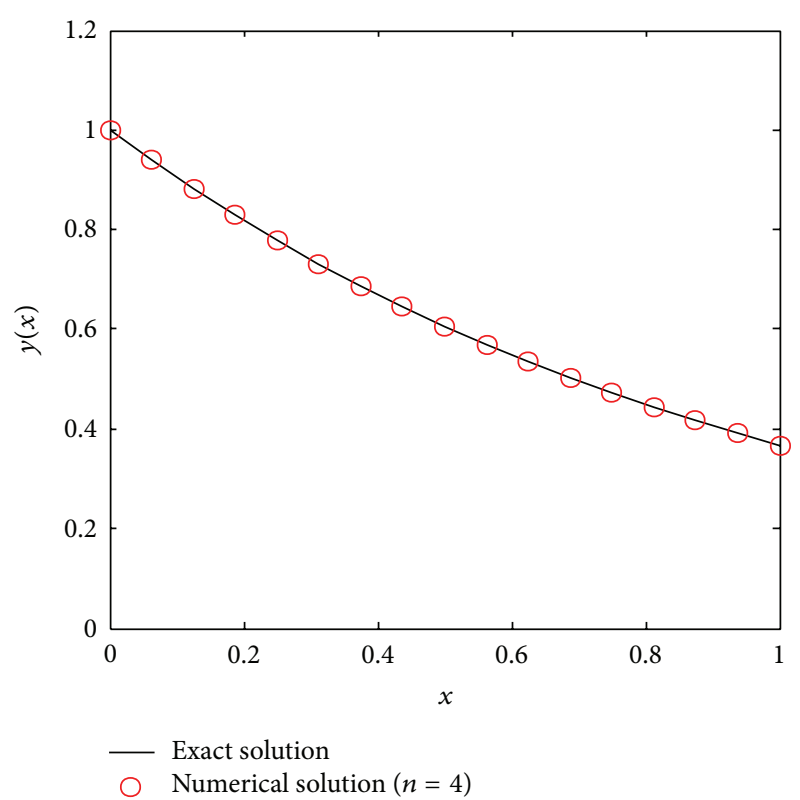

FIGURE 1: Comparison between the numerical and exact solution for Example 1.

TABLE 1: Absolute errors for Example 1, $y(x)=e^{-x}$.

\begin{tabular}{lccc}
\hline$x$ & $n=3$ & $n=4$ & $n=5$ \\
\hline 0.1250 & $7.6669386 E-09$ & $4.1229653 E-10$ & $1.7578161 E-12$ \\
0.2500 & $8.7825011 E-09$ & $1.2445001 E-09$ & $6.3016259 E-13$ \\
0.3750 & $4.7608193 E-09$ & $8.1603835 E-09$ & $3.4359182 E-12$ \\
0.5000 & $1.1092671 E-09$ & $1.9748597 E-10$ & $6.4426242 E-13$ \\
0.6750 & $46921567 E-09$ & $2.783539 E-10$ & $1.3058443 E-12$ \\
0.7500 & $1.1643215 E-08$ & $3.4349251 E-10$ & $1.4662715 E-12$ \\
0.8750 & $1.8162271 E-09$ & $5.5568805 E-10$ & $1.3625767 E-12$ \\
1.0000 & $1.110223 E-16$ & $2.9802344 E-10$ & $5.5511151 E-17$ \\
\hline
\end{tabular}

Example 2. Consider a nonlinear Volterra integral equation with continuous kernel [26]

$$
y(x)=f(x)+\int_{0}^{x} x t^{2}[y(t)]^{2} d t
$$

where $f(x)=\left(1-11 / 9 x+2 / 3 x^{2}-1 / 3 x^{3}+2 / 9 x^{4}\right) \ln (x+1)-$ $1 / 3\left(x+x^{4}\right)(\ln (x+1))^{2}-11 / 9 x^{2}+5 / 18 x^{3}-2 / 27 x^{4}$.

The exact solution is $y(x)=\ln (x+1)$.

Figure 2 shows the comparison between the exact result and the numerical result for $n=4$. It can be seen that the solution is convergent; even though the number of nodes is sixteen $(n=4)$, it is nearly the exact solution. The absolute errors for Example 2 when $n=3,4,5$ are given in Table 2. It can be found that the present results with 8 grid points are much more accurate than the results given by the method with 8 grid points [26].

Example 3. Consider the following Fredholm integral equation [26]:

$$
y(x)=e^{x}-\frac{\left(1+2 e^{3}\right) x}{9}+\int_{0}^{1} x t[y(t)]^{3} d t .
$$




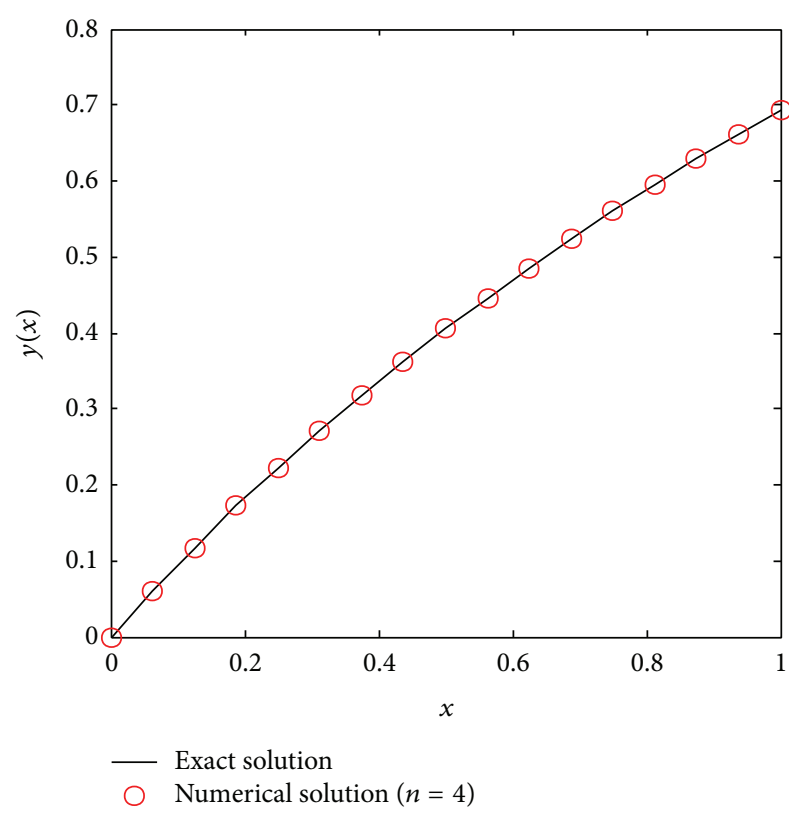

FIGURE 2: Comparison between the numerical and exact solution for Example 2.

TABLE 2: Absolute errors for Example 2, $y(x)=\ln (x+1)$.

\begin{tabular}{lccc}
\hline$x$ & $n=3$ & $n=4$ & $n=5$ \\
\hline 0.1250 & $1.0550211 E-06$ & $3.5971851 E-08$ & $1.3776 E-09$ \\
0.2500 & $1.6221136 E-06$ & $7.3412183 E-08$ & $2.7710071 E-09$ \\
0.3750 & $2.5592494 E-06$ & $1.1107313 E-07$ & $4.1670886 E-09$ \\
0.5000 & $3.3524516 E-06$ & $1.4938059 E-07$ & $5.6009465 E-09$ \\
0.6750 & $4.3063136 E-06$ & $1.9062777 E-07$ & $7.1452912 E-09$ \\
0.7500 & $5.3589701 E-06$ & $2.3888217 E-07$ & $8.9525424 E-09$ \\
0.8750 & $6.7969976 E-06$ & $3.010169 E-07$ & $1.1321046 E-08$ \\
1.0000 & $7.8925395 E-06$ & $3.8939399 E-07$ & $1.4587509 E-08$ \\
\hline
\end{tabular}

One can easily find that the exact solution of this equation is $y(x)=e^{x}$. Figure 3 shows that the approximation solution obtained by the present method has a good agreement with the exact solution. The corresponding absolute errors for different $n$ are given in Table 3 .

\section{Conclusions}

In this paper, by combining techniques of boundary extension and Coiflet-type wavelet expansion, an approximation scheme of the function defined on a finite interval is proposed. With such a modified Coiflet-type wavelet approximation scheme, any nonlinear term containing unknown function can be explicitly expressed by a single-point sampling of the function successfully. Using Galerkin method, finally, based on this method, the original nonlinear integral equation was transformed into the solving of some simple nonlinear algebraic equations. Since it does not need to calculate the connection coefficients, it avoids numerical errors and reduces the computational complexity of

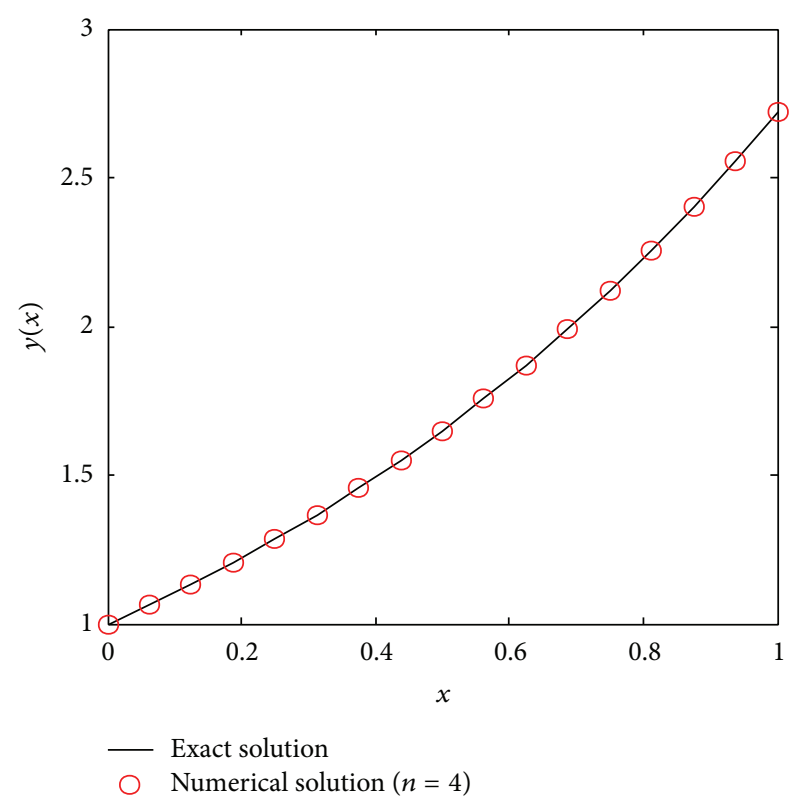

FIGURE 3: Comparison between the numerical and exact solution for Example 3.

TABLE 3: Absolute errors for Example 3, $y(x)=e^{x}$.

\begin{tabular}{lccc}
\hline$x$ & $n=3$ & $n=4$ & $n=5$ \\
\hline 0.1250 & $4.5667344 E-05$ & $1.7712256 E-06$ & $6.2164018 E-08$ \\
0.2500 & $9.1334688 E-05$ & $3.5424513 E-06$ & $1.2432804 E-07$ \\
0.3750 & $1.3700203 E-04$ & $5.3136783 E-06$ & $1.8649594 E-07$ \\
0.5000 & $1.8266938 E-04$ & $7.0849024 E-06$ & $2.4865602 E-07$ \\
0.6750 & $2.2833672 E-04$ & $8.8561295 E-06$ & $3.1082206 E-07$ \\
0.7500 & $2.7400406 E-04$ & $1.0627356 E-05$ & $3.7299193 E-07$ \\
0.8750 & $3.1967141 E-04$ & $1.2398584 E-05$ & $4.3515077 E-07$ \\
1.0000 & $3.6533875 E-04$ & $1.4169805 E-05$ & $4.9731233 E-07$ \\
\hline
\end{tabular}

the connection coefficients. Moreover, numerical errors of the present method are not sensitive to the nonlinear intensity of the equations. Also in the future, the method proposed in this paper is expected to be further applied to solve other nonlinear problems in other fields.

\section{Conflict of Interests}

The author declares that there is no conflict of interests regarding the publication of this paper.

\section{Acknowledgments}

The work was supported by the National Natural Science Foundation of China (11302081) and Huazhong Agricultural University Scientific and Technological Self-Innovation Foundation under Grant 52902-0900206074. 


\section{References}

[1] F. Bloom, "Asymptotic bounds for solutions to a system of damped integro-differential equations of electromagnetic theory," Journal of Mathematical Analysis and Applications, vol. 73, no. 2, pp. 524-542, 1980.

[2] M. A. Abdou, "Fredholm-Volterra integral equation of the first kind and contact problem," Applied Mathematics and Computation, vol. 125, no. 2-3, pp. 177-193, 2002.

[3] J. I. Frankel, "A Galerkin solution to a regularized Cauchy singular integro-differential equation," Quarterly of Applied Mathematics, vol. 53, no. 2, pp. 245-258, 1995.

[4] K. Y. Wang and Q. S. Wang, "Taylor collocation method and convergence analysis for the Volterra-Fredholm integral equations," Journal of Computational and Applied Mathematics, vol. 260, pp. 294-300, 2014.

[5] H. Brunner, A. Makroglou, and R. K. Miller, "On mixed collocation methods for Volterra integral equations with periodic solution," Applied Numerical Mathematics, vol. 24, no. 2-3, pp. 115-130, 1997.

[6] S. Yalcinba and M. Sezer, "A method for the approximate solution of the second-order linear differential equations in terms of Taylor polynomials," vol. 27, pp. 821-834, 1996.

[7] A. Karamete and M. Sezer, "A Taylor collocation method for the solution of linear integro-differential equations," International Journal of Computer Mathematics, vol. 79, no. 9, pp. 987-1000, 2002.

[8] S. Yalçinbaş and M. Sezer, "A Taylor collocation method for the approximate solution of general linear Fredholm-Volterra integro-difference equations with mixed argument," Applied Mathematics and Computation, vol. 175, no. 1, pp. 675-690, 2006.

[9] E. Babolian, S. Abbasbandy, and F. Fattahzadeh, "A numerical method for solving a class of functional and two dimensional integral equations," Applied Mathematics and Computation, vol. 198, no. 1, pp. 35-43, 2008.

[10] E. Babolian and A. Shahsavaran, "Numerical solution of nonlinear Fredholm integral equations of the second kind using Haar wavelets," Journal of Computational and Applied Mathematics, vol. 225, no. 1, pp. 87-95, 2009.

[11] X. Z. Liang, M. C. Liu, and X. J. Che, "Solving second kind integral equations by Galerkin methods with continuous orthogonal wavelets," Journal of Computational and Applied Mathematics, vol. 136, no. 1-2, pp. 149-161, 2001.

[12] J. Saberi-Nadjafi, M. Mehrabinezhad, and T. Diogo, "The Coiflet-Galerkin method for linear Volterra integral equations," Applied Mathematics and Computation, vol. 221, pp. 469-483, 2013.

[13] Y. H. Ren, B. Zhang, and H. Qiao, "A simple Taylor-series expansion method for a class of second kind integral equations," Journal of Computational and Applied Mathematics, vol. 110, no. 1, pp. 15-24, 1999.

[14] M. T. Rashed, "Lagrange interpolation to compute the numerical solutions of differential, integral and integro-differential equations," Applied Mathematics and Computation, vol. 151, no. 3, pp. 869-878, 2004.

[15] K. Wang and Q. Wang, "Lagrange collocation method for solving Volterra-Fredholm integral equations," Applied Mathematics and Computation, vol. 219, no. 21, pp. 10434-10440, 2013.

[16] Y. P. Chen and T. Tang, "Spectral methods for weakly singular Volterra integral equations with smooth solutions," Journal of
Computational and Applied Mathematics, vol. 233, no. 4, pp. 938-950, 2009.

[17] X. Liu, J. Wang, X. Wang, and Y. Zhou, "Exact solutions of multi-term fractional diffusion-wave equations with Robin type boundary conditions," Applied Mathematics and Mechanics (English Edition), vol. 35, no. 1, pp. 49-62, 2014.

[18] Y. H. Zhou, X. M. Wang, J. Z. Wang, and X. J. Liu, "A wavelet numerical method for solving nonlinear fractional vibration, diffusion and wave equations," Computer Modeling in Engineering \& Sciences, vol. 77, no. 2, pp. 137-160, 2011.

[19] A. Avudainayagam and C. Vani, "Wavelet-galerkin method for integro-differential equations," Applied Numerical Mathematics, vol. 32, no. 3, pp. 247-254, 2000.

[20] Y. H. Zhou and J. Z. Wang, "A generalized Gaussian integral method for the calculation of scaling function transforms of wavelets and its applications," Acta Mathematica Scientia, vol. 19, no. 3, pp. 293-300, 1999.

[21] J. Wang, Y. Zhou, and H. Gao, "Computation of the Laplace inverse transform by application of the wavelet theory," Communications in Numerical Methods in Engineering, vol. 19, no. 12, pp. 959-975, 2003.

[22] Y. Zhou, J. Wang, X. J. Zheng, and Q. Jiang, "Vibration control of variable thickness plates with piezoelectric sensors and actuators based on wavelet theory," Journal of Sound and Vibration, vol. 237, no. 3, pp. 395-410, 2000.

[23] J. Wang, X. Wang, and Y. Zhou, "A wavelet approach for activepassive vibration control of laminated plates," Acta Mechanica Sinica, vol. 28, no. 2, pp. 520-531, 2012.

[24] M. T. Rashed, "Numerical solution of functional differential, integral and integro-differential equations," Applied Mathematics and Computation, vol. 156, no. 2, pp. 485-492, 2004.

[25] J. Z. Wang, Generalized theory and arithmetic of orthogonal wavelets and applications to researches of mechanics including piezoelectric smart structures [Ph.D dissertation], Lanzhou University, Lanzhou, China, 2001.

[26] Y. Mahmoudi, "Wavelet Galerkin method for numerical solution of nonlinear integral equation," Applied Mathematics and Computation, vol. 167, no. 2, pp. 1119-1129, 2005. 


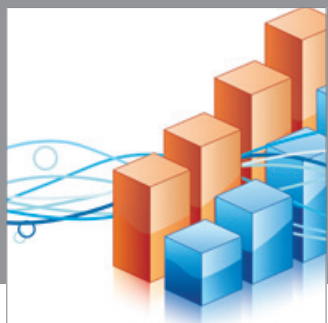

Advances in

Operations Research

mansans

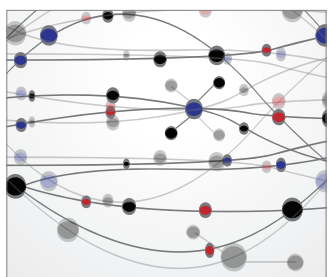

The Scientific World Journal
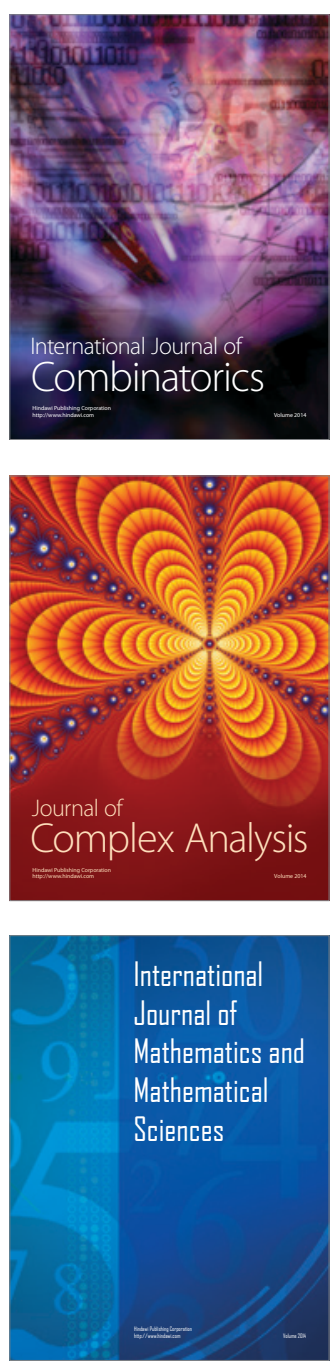
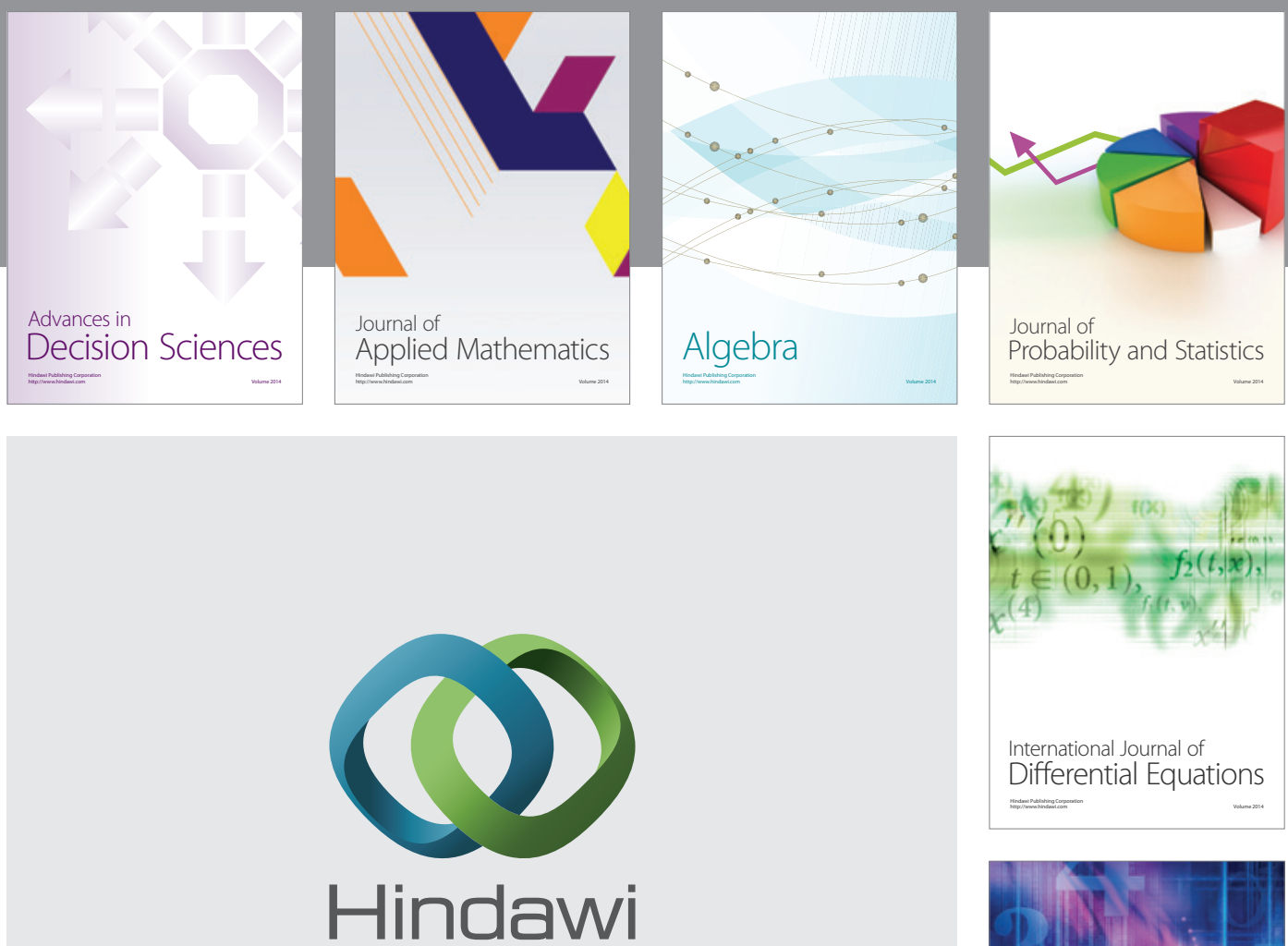

Submit your manuscripts at http://www.hindawi.com
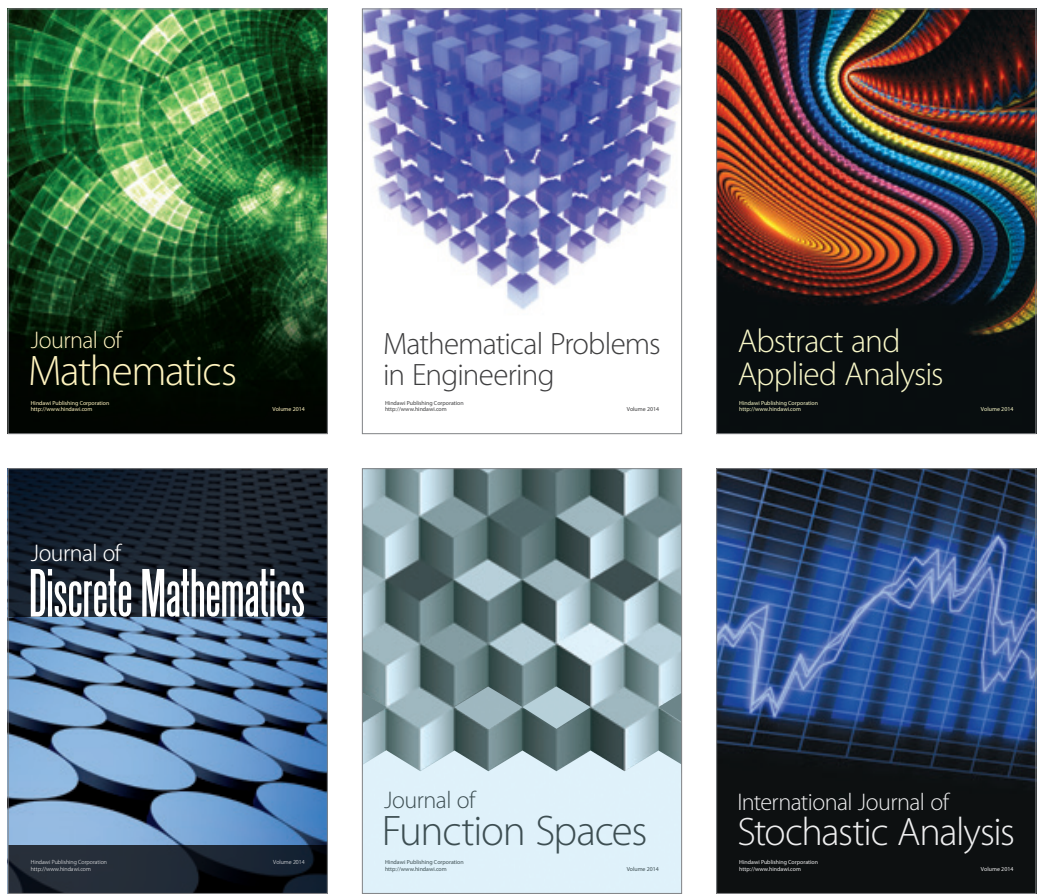

Journal of

Function Spaces

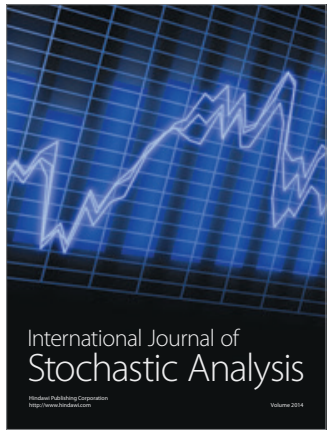

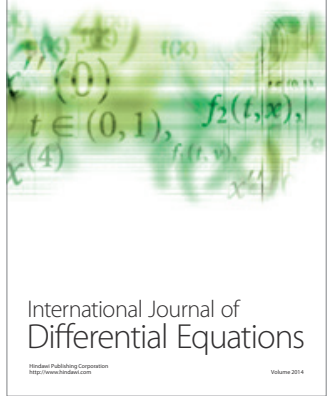
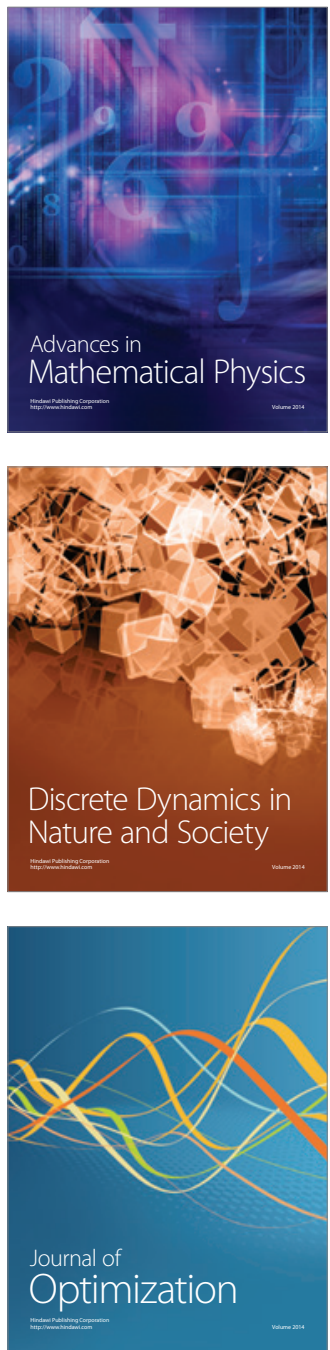